\title{
Superficial brachial artery: a possible cause for idiopathic median nerve entrapment neuropathy
}

\author{
P. Nkomozepi, N. Xhakaza, E. Swanepoel \\ Department of Human Anatomy and Physiology, Faculty of Health Sciences, University of Johannesburg, \\ Johannesburg, Gauteng, South Africa
}

[Received: 29 November 2016; Accepted: 4 January 2017]

\begin{abstract}
Nerve entrapment syndromes occur because of anatomic constraints at specific locations in both upper and lower limbs. Anatomical locations prone to nerve entrapment syndromes include sites where a nerve courses through fibro-osseous or fibromuscular tunnels or penetrates a muscle. The median nerve $(M N)$ can be entrapped by the ligament of Struthers; thickened biceps aponeurosis; between the superficial and deep heads of the pronator teres muscle and by a thickened proximal edge of flexor digitorum superficialis muscle. A few cases of $\mathrm{MN}$ neuropathies encountered are reported to be idiopathic. The superficial branchial artery (SBA) is defined as the artery running superficial to $M N$ or its roots. This divergence from normal anatomy may be the possible explanation for idiopathic MN entrapment neuropathy.

This study presents three cases with unilateral presence of the SBA encountered during routine undergraduate dissection at the University of Johannesburg. Case 1 — SBA divided into radial and ulnar arteries. Brachial artery (BA) terminated as deep brachial artery. Case 2 - SBA continued as radial artery (RA). BA terminated as ulnar artery (UA), anterior and posterior interosseous arteries. Case $3-S B A$ continued as UA. BA divided into radial and common interosseous arteries.

Arteries that take an unusual course are more vulnerable to iatrogenic injury during surgical procedures and may disturb the evaluation of angiographic images during diagnosis. In particular, the presence of SBA may be a course of idiopathic neuropathies. (Folia Morphol 2017; 76, 3: 527-531)
\end{abstract}

Key words: superficial brachial artery, median nerve entrapment, entrapment neuropathies

\section{INTRODUCTION}

Entrapment neuropathies are characterised by alterations of nerve function that are caused by mechanical or dynamic compression. Nerve entrapment syndromes occur because of anatomic constraints at specific locations in both upper and lower limbs [2]. Anatomical locations, that are prone to nerve entrapment syndromes, include sites where a nerve 1) courses through fibro-osseous or fibromuscular tunnels or 2) penetrates a muscle [17].

The median nerve (MN) is formed in the axilla, lateral to the axillary artery, by the union of its medial and lateral roots originating respectively from the medial and lateral cords of the brachial plexus. The nerve's lateral root crosses to the medial aspect, superficial to the third part of the axillary artery, to

Address for correspondence: Mr. P. Nkomozepi, Department of Human Anatomy and Physiology, Faculty of Health Sciences, University of Johannesburg, cnr Beit and Sherwell streets, Johannesburg, Gauteng, South Africa, tel: +2711559 6722, e-mail: pilanin@uj.ac.za 


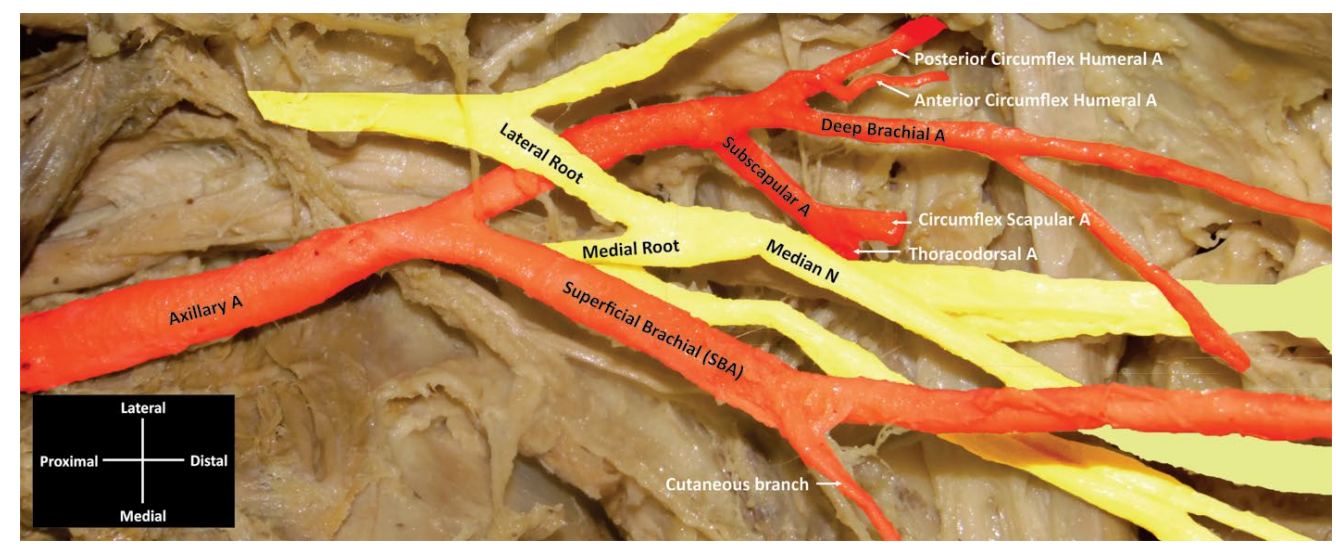

Figure 1. Anterior view of the left upper limb of a 75-year-old male Caucasian showing a superficial brachial artery (SBA).

meet the medial root where the MN is formed. In the arm, the MN crosses superficial to the brachial artery from lateral to medial and enters the cubital fossa along with the brachial [13].

Median nerve entrapment syndromes have mostly been reported to occur at points between the elbow and the wrist joints. The MN can be entrapped in the lower part of the arm at the distal part of the humerus by the ligament of Struthers; proximal elbow by a thickened biceps aponeurosis; elbow joint between the superficial and deep heads of the pronator teres muscle and proximal forearm by a thickened proximal edge of the flexor digitorum superficialis $[2,4,11,18]$.

Adachi (1928) [1] defined the superficial branchial artery (SBA) as the artery that runs superficial to the MN. When present, the SBA may replace the main brachial artery trunk completely or may be accompanied by an equally important, more important or less important brachial artery trunk running deep to the MN [10]. This slight divergence from normal anatomy in the axilla may result in $\mathrm{MN}$ entrapment [2].

A few cases of MN neuropathies encountered in the clinical setting are reported to be idiopathic [15]. Entrapment of the MN or its roots between the SBA and the axillary artery could be a possible culprit in idiopathic MN neuropathies. In this article we present three cases with unilateral presence of the SBA that were encountered during routine undergraduate dissection at the University of Johannesburg and investigated as part of a larger study regarding upper limb arterial variations which received ethical clearance from the Faculty of Health Sciences Academic Ethics committee.

\section{CASE 1}

The SBA was present in the left upper limb of a 75-year-old male Caucasian (Fig. 1).

The axillary artery gave off a SBA which descended superficial to the medial root of the MN and gave rise to a cutaneous branch that supplied the skin on the floor of the axilla. It terminated by bifurcating into radial and ulnar arteries in the cubital fossa. The axillary artery trunk passed deep to the lateral root of the MN, gave off subscapular artery, anterior and posterior circumflex humeral arteries and terminated as the deep brachial artery (Fig. 1).

\section{CASE 2}

The SBA was present in the left upper limb of a 63-year-old female Caucasian (Fig. 2A, B).

The SBA originated from the axillary artery, descended superficial to the medial root of the MN (Fig. 2A), crossed the cubital fossa superficial to the biceps aponeurosis and continued as the radial artery in the forearm (Fig. 2B). The axillary artery trunk descended posterior to the lateral root of the $\mathrm{MN}$, became the brachial artery in the arm, descended deep to the MN and terminated by branching into the ulnar, anterior interosseous and posterior interosseous arteries as well as arterial muscular branches in the cubital fossa (Fig. 2B).

\section{CASE 3}

The SBA was present in the right upper limb of an 88-year-old female Caucasian.

The SBA arose from the second part of the axillary artery, crossed superficial to the medial root of the $\mathrm{MN}$ and continued superficially into the forearm where it became continuous with the ulnar artery 


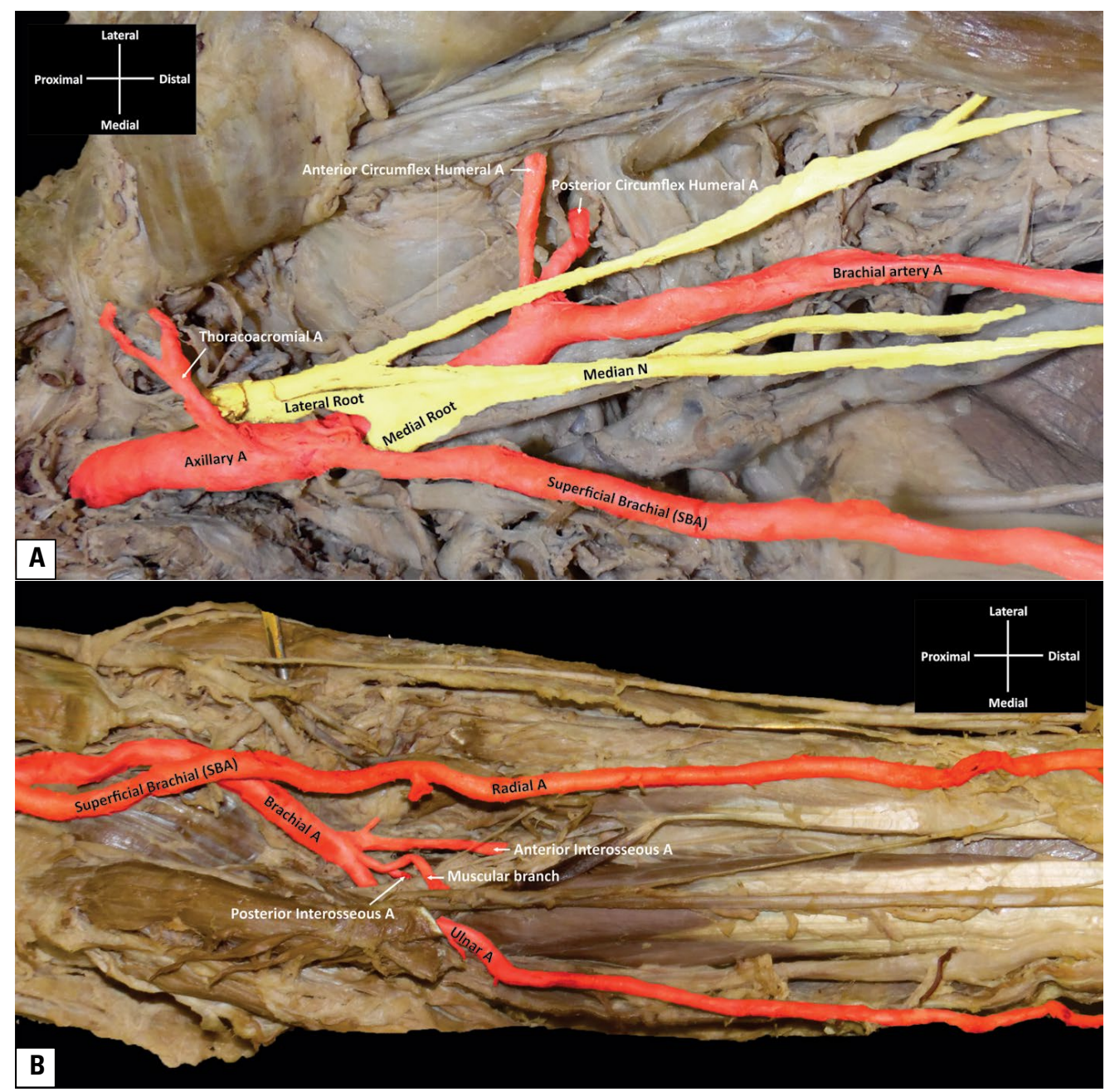

Figure 2. A. Anterior view of the left upper limb of a 63-year-old female Caucasian showing a superficial brachial artery (SBA); B. Anterior view of the left upper limb of a 63-year-old female Caucasian showing the continuation of a SBA and the divisions of the related brachial artery.

(Fig. 3A). The axillary artery trunk descended deep to the lateral root of the MN and divided into the radial and common interosseous arteries in the cubital fossa (Fig 3B).

\section{DISCUSSION}

Variations in the arterial pattern of the upper limb are common and have been reported by several authors. The term SBA was first defined in 1928 as that artery that runs superficial to the MN [1]. The incidence of the SBA in the different populations studied ranged from $0.12 \%$ to $19.7 \%$ [10].

Yang et al. [19] classified the SBA into three types: Type I: the SBA bifurcated into the radial and ulnar arteries in the cubital fossa after giving off muscular branches to the biceps brachii and brachialis muscles. In this type, the anterior and posterior interosseous arteries arose either separately or as a common trunk from the ulnar artery. The ulnar artery coursed posterior to the flexor carpi ulnaris muscle. The axillary artery continued as the traditional brachial artery which terminated as a collateral branch around the elbow [19]. This type corresponds to Case 1 of the current study. However, in the present study, the brachial artery did not terminate as a collateral branch but rather terminated as the deep brachial artery which accompanied the radial nerve into the radial groove. Previous studies have reported the prevalence of the deep brachial artery originating from the axillary artery as follows (in increasing order of prevalence): $2 \%$ [14]; 8\% [7]; 8.57\% [5]; 8.7\% [6]; and 16.6\% [3]. Type II SBA continued as the radial artery after branching into muscular branches to the biceps brachii and brachialis muscles. This type corresponds to Case 2 


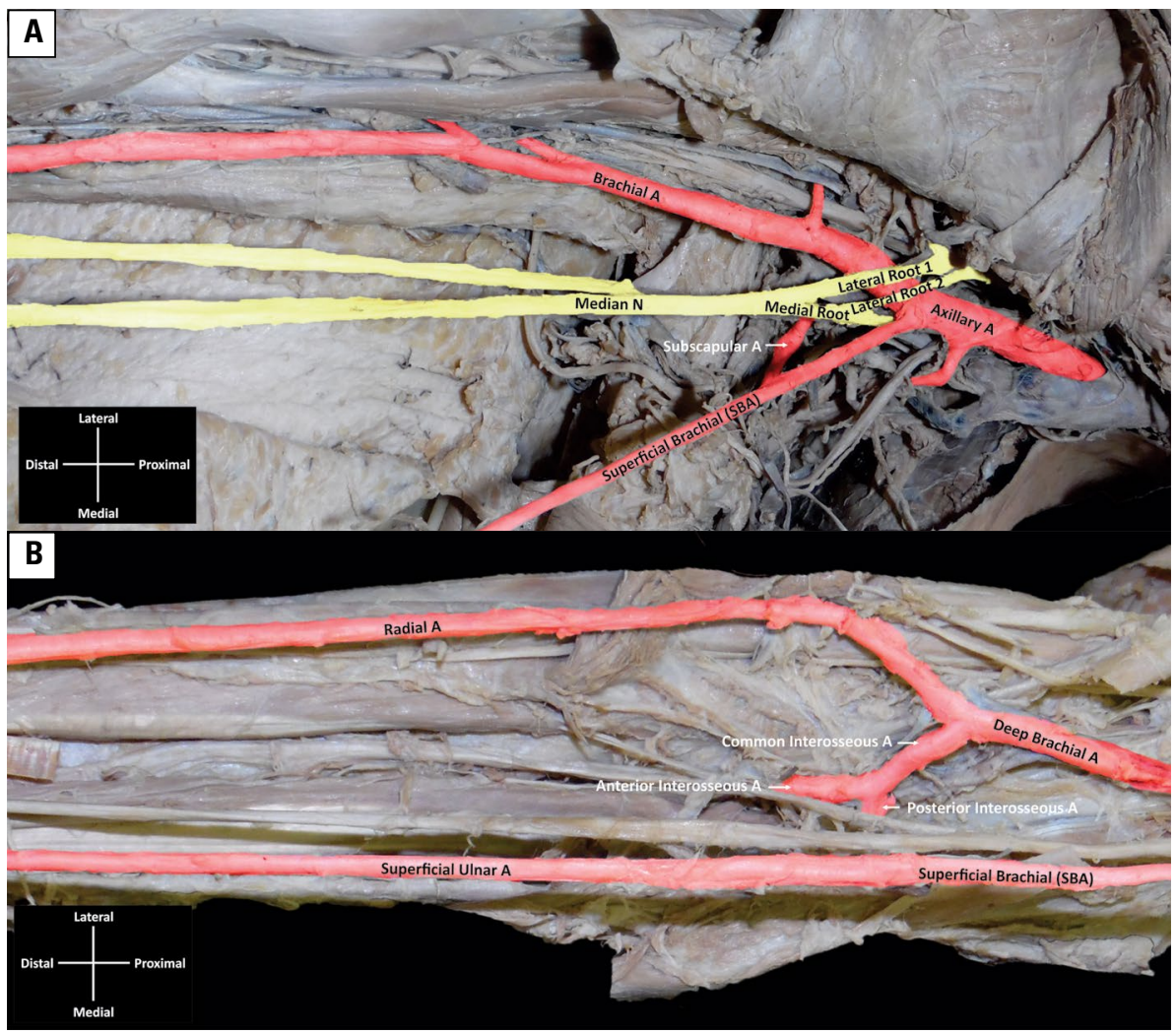

Figure 3. A. Anterior view of the right upper limb of an 88-year-old female Caucasian showing a superficial brachial artery (SBA); B. Anterior view of the right upper limb of an 88-year-old female Caucasian showing a SBA and divisions of the related brachial artery.

of the present study. In Type III, the slender SBA supplied the arm musculature and terminated in the arm.

The SBA that continued into the ulnar side of the hand as recorded in Case 3 of the present study could not be classified according to Yang et al.'s [19] classification. However, an example similar to Case 3 was reported by Chakravarthi et al. (2014) [5] in their study of 140 upper limbs. The authors referred to the continuation of the SBA in the forearm as the accessory ulnar artery. They found this pattern in three cadavers, $4.29 \%$ cases.

The SBA may be a potential cause of MN compression which may help explain the cause of idiopathic MN neuropathies. Its presence can be best understood by revisiting the normal development of the upper limb arterial pattern as described by Singer (1933) [16]. The SBA first appears in the $21 \mathrm{~mm}$ long embryo at stage IV. The SBA develops in the region of the axilla and traverses the medial aspect of the arm. It descends diagonally from the ulnar side to the radial side of the forearm to reach the posterior surface of the wrist joint. Here the SBA divides over the carpus into branches for the dorsal aspect of the thumb and index finger [16].

At Singer's stage $V$, following retrogression of the median artery, the SBA gives off a distal branch which anastomoses with the superficial palmar arch. Near the elbow joint, an anastomotic branch between the brachial artery and the SBA becomes enlarged and joins the distal portion of the brachial artery to form the radial artery. The proximal portion of the SBA joining the axillary artery to the radial artery retrogresses. Case 1 in the current study corresponds to the case reported by Khullar (2014) [10] where the SBA terminated by dividing into radial and ulnar arteries, the deep brachial artery terminating by giving twigs to the arm. A similar case was also reported by Janaki et al. (2016) [9]; however, in their case, the deep brachial artery did not terminate in the arm, but continued to the cubital fossa as the common interosseous artery which divided into anterior and posterior interosseous artery. Therefore, on an embryological basis, this suggests that in stage III of Singer's theory, the brachial artery (between origin of SBA and ulnar artery) retrogressed and lost its com- 
munication with the common interosseous artery. The SBA thus persisted and continued as the radial artery. In Case 2 (present study), the proximal part of SBA in stage $V$ of Singer's theory may have failed to retrogress and continued as the radial artery and an anastomotic branch between the SBA and brachial artery may have failed to form. In Case 3 (current study), it seems in stage IV of Singer's theory, the SBA originated from the axillary artery, but did not cross diagonally to the radial side, but rather continued as the superficial ulnar artery. A similar case was recorded by Nakatani et al. [12].

Arteries that take an unusual course are more vulnerable to iatrogenic injury during surgical procedures and may disturb the evaluation of angiographic images during diagnosis [8]. In particular, the presence of SBA may be a cause of idiopathic neuropathies.

\section{Acknowledgements}

The authors would like to thank the persons who selflessly donated their bodies in the pursuit of furthering education and research.

\section{REFERENCES}

1. Adachi B. Das Arteriensystem der Japaner. Maruzen Press, Kyoto 1928.

2. Andreisek G, Crook DW, Burg D, et al. Peripheral neuropathies of the median, radial, and ulnar nerves: MR imaging features. Radiographics. 2006; 26(5): 1267-1287, doi: 10.1148/rg.265055712, indexed in Pubmed: 16973765.

3. Anson BJ. The Cardiovascular system - Arteries \& Veins. In: Thomas M (ed) Morris Hum. Anat. McGraw Hill Book Company, New York 1966: 708-724.

4. Bilecenoglu B, Uz A, Karalezli N. Possible anatomic structures causing entrapment neuropathies of the median nerve: an anatomic study. Acta Orthop Belg. 2005; 71(2): 169-176, indexed in Pubmed: 16152850.

5. Chakravarthi KK, Ks S, Venumadhav N, et al. Anatomical variations of brachial artery - its morphology, embryogenesis and clinical implications. J Clin Diagn Res. 2014; 8(12): AC17-AC20, doi: 10.7860/JCDR/2014/10418.5308, indexed in Pubmed: 25653931.
6. Charles CM, Penn L, Holden HF, et al. The origin of the deep brachial artery in American white and in American negro males. Anat Rec. 1931; 50(3): 299-302, doi: 10.1002/ ar.1090500307.

7. Chauhan K, Udainia A, Bhatt C, et al. Morphological study of variation in branching pattern of brachial artery. Int J Basic Appl Med Sci. 2013; 3: 10-15.

8. Gonzalez-Compta X. Origin of the radial artery from the axillary artery and associated hand vascular anomalies. J Hand Surg Am. 1991; 16(2): 293-296, indexed in Pubmed: 2022840.

9. Janaki V, Sumalatha T, Veenatai J. Aparna Vedapriya K Variant Course and Anamolous branching Pattern of Major Ateries In Upper Limb in Telangana Region. Indian J Appl Res. 2016; 6: 103-106.

10. Khullar M. Superficial brachial artery: its embryological and clinical significance. Indian J Clin Pract. 2014; 24: 925-928.

11. Lee MJ, LaStayo PC. Pronator syndrome and other nerve compressions that mimic carpal tunnel syndrome. J Orthop Sports Phys Ther. 2004; 34(10): 601-609, doi: 10.2519/ jospt.2004.34.10.601, indexed in Pubmed: 15552706.

12. Nakatani T, Tanaka S, Mizukami S, et al. The superficial ulnar artery originating from the axillary artery. Ann Anat. 1996; 178(3): 277-279, doi: 10.1016/S09409602(96)80068-9, indexed in Pubmed: 8712378.

13. Nandi-Kar M. Kar Proximal entrapment of median nerve associated with axillary artery variation: a case report. Case Rep Int J Anat Var. 2013; 6: 188-190.

14. Patnaik V, Kalsey G. Singla Rajan K Branching pattern of brachial artery: a morphological study. J Anat Soc India. 2002; 51: 176-186.

15. Rosenbaum RB, Ochoa J. Carpal Tunnel Syndrome and Other Disorders of the Median Nerve, 2e, 2 edition. Butterworth-Heinemann, Woburn, MA 2002.

16. Singer E. Embryological pattern persisting in the arteries of the arm. Anat Rec. 1933; 55(4): 406-413, doi: 10.1002/ ar.1090550407.

17. Spinner RJ, Amadio PC. Compressive neuropathies of the upper extremity. Clin Plast Surg. 2003; 30(2): 155-73, vi, doi: 10.1016/S0094-1298(02)00103-7, indexed in Pubmed: 12737350.

18. Tsai P, Steinberg DR. Median and radial nerve compression about the elbow. Instr Course Lect. 2008; 57: 177-185, indexed in Pubmed: 18399579.

19. Yang HJ, Gil YC, Jung WS, et al. Variations of the superficial brachial artery in Korean cadavers. J Korean Med Sci. 2008; 23(5): 884-887, doi: 10.3346/jkms.2008.23.5.884, indexed in Pubmed: 18955798. 